\title{
Enfermedad renal crónica: clasificación, mecanismos de progresión y estrategias de renoprotección
}

\author{
Sergio Mezzano A, Claudio Aros E. \\ Chronic kidney disease: \\ Classification, mechanisms of \\ progression and strategies for \\ renoprotection
}

Chronic kidney disease is a worldwide health problem. The incidence and prevalence of kidney failure is in constant increase, involving poor outcomes and high costs. The leading causes of kidney failure are type 2 diabetes and hypertension. The new initiative "Kidney Disease Improving Global Outcomes (KDIGO)" is a global public health approach to face this problem. A formal definition for chronic kidney disease and a staging of kidney diseases from kidney damage with preserved function to kidney failure, were proposed. We reviewed the main mechanisms involved in renal disease progression, with emphasis in the proteinuria and the intrarenal activation of renin angiotensin system. Moreover, the evidence in the literature of therapeutical interventions with proved efficacy in slowing the rate of reduction of renal function is discussed, particularly the optimal control of hypertension, reduction of proteinuria and renin angiotensin system blocking. Finally, we recommend a strategy for the clinical management of patients in the different stages of chronic kidney disease (Rev Méd Chile 2005; 133: 338-48).

(Key Words: Diabetic nephropathy; Glomerular filtration rate; Kidney diseases; Kidney failure; Proteinuria)

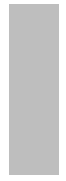

\footnotetext{
Artículo preparado por invitación de los Editores. Recibido el 17 de diciembre, 2004. Aceptado el 14 de enero, 2005. Proyecto FONDECYT \# 1040163.

Unidad de Nefrología, Instituto de Medicina, Facultad de Medicina, Universidad Austral de Chile, Valdivia, Chile.
}

Correspondencia a: Dr. Sergio Mezzano A. Laboratorio de Nefrología, Universidad Austral de Chile. Servicio de Medicina, Hospital Clínico Regional Valdivia. Simpson 850, Valdivia, Chile. Fono-Fax: ++56 63 215890. E mail: smezzano@uach.cl 
L a enfermedad renal crónica ha adquirido las proporciones de una verdadera epidemia, cuyo espectro completo recién comienza a entenderse. En los Estados Unidos de Norteamérica, el número de pacientes con insuficiencia renal crónica terminal (IRCT), que requieren tratamiento sustitutivo renal, ha aumentado más de tres veces en las últimas dos décadas, llegando a una incidencia de 334 pacientes por millón de habitantes. Considerando la tendencia demográfica, se ha proyectado que en el año 2030, habrá aproximadamente 2,2 millones de pacientes que requerirán diálisis o trasplante ${ }^{1}$ (Figura 1).

Dentro de las causas que llevan a la insuficiencia renal crónica, también en Estados Unidos, la diabetes tipo 2 ocupa el primer lugar dando cuenta de $40 \%$ de los pacientes que ingresan a tratamiento sustitutivo renal. Paralelo al aumento explosivo de los costos, se espera que la incidencia mundial de IRCT entre los diabéticos tipo 2 se habrá duplicado para el año $2010^{2}$. Tendencias similares se han comunicado en otras regiones.

Además de los pacientes con IRCT, se ha estimado que al menos 8 millones de norteamericanos tienen una velocidad de filtración glomerular (VFG) disminuida en grado leve a moderado (fases 3 y 4 , VFG $30-59$ y $15-29$ mL/min/ $1,73 \mathrm{~m}^{2}$ respectivamente) y, además, la presencia de enfermedad renal crónica es clínicamente importante no sólo porque puede progresar a IRCT o fase 5, correspondiente a una VFG menor de $15 \mathrm{~mL} / \mathrm{min} / 1,73 \mathrm{~m}^{2}$, sino también, porque se asocia independientemente a un aumento de la morbimortalidad cardiovascular ${ }^{1}$. En un estudio reciente, en el cual se estimó la VFG en una población superior a un millón de personas, se observó que para valores inferiores a $60 \mathrm{~mL}$ el riesgo de muerte, de eventos cardiovasculares y de hospitalizaciones aumenta paralelamente con la disminución del filtrado glomerular. Estos hallazgos confirman la importancia en clínica y en la salud pública de la enfermedad renal crónica $(\mathrm{ERC})^{3}$.

En Chile, la prevalencia de la ERC ha sido estudiada recientemente en el marco del Proyecto Encuesta Nacional de Salud 2003, del Ministerio de Salud. Se observó que la prevalencia de ERC en fases 3 y 4 (VFG de 30-59 y 15-29 mL respectivamente) es 5,7\% y 0,2\%, respectivamen$t^{4}$. Estos porcentajes aumentan significativamente en mayores de 45 años y particularmente en mayores de 65 años. Adicionalmente, de acuerdo a lo comunicado en la Encuesta de Diálisis en Chile, si consideramos sólo los pacientes en hemodiálisis, la prevalencia de ERC en fase 5 correspondería aproximadamente a 0,1\%.

No existiendo curación definitiva para la mayor parte de las ERC, y estando el trasplante renal seriamente limitado por la disponibilidad de órganos, la mejor estrategia en el momento actual es concentrar nuestros esfuerzos en el conocimiento

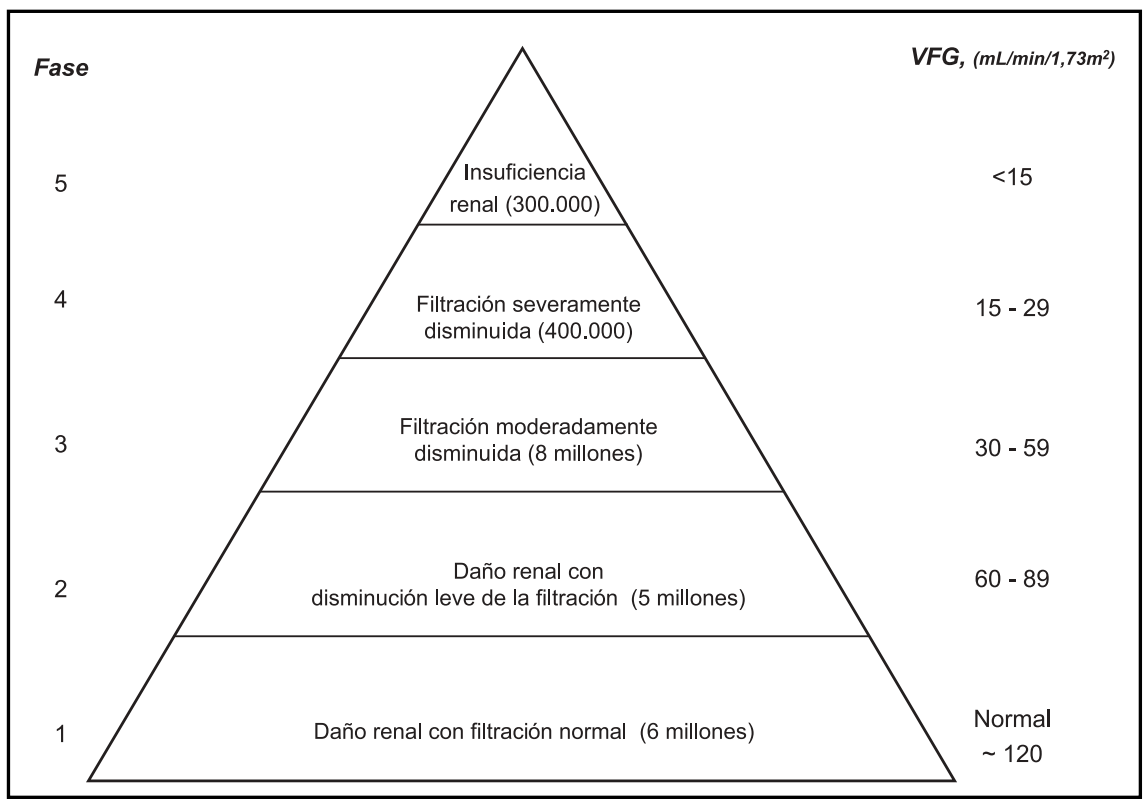

Figura 1. Prevalencia actual de la enfermedad renal crónica en sus diferentes fases según el U.S. Renal Data System (Ref 1). 
de los mecanismos moleculares de la progresión y en las estrategias de prevención de la progresión (renoprotección).

\section{CONCEPTO DE ENFERMEDAD RENAL CRÓNICA Y ETAPIFICACIÓN}

Actualmente, las principales causas de ERC son en primer lugar la diabetes y luego la hipertensión arterial, que en conjunto representan aproximadamente $60 \%$ de los pacientes en diálisis crónica. En Chile se ha estimado, en base a los datos de la Encuesta Nacional de Salud 2003, que la prevalencia de diabetes es de 4,2\% y la de hipertensión arterial de $33,7 \%{ }^{4}$.

En relación a la ERC se han usado una serie de términos que muchas veces han causado confusión. Con el fin de introducir una terminología uniforme, la National Kidney Foundation (NKFUSA), en su Iniciativa para la Mejoría de los Resultados Globales en Enfermedades Renales (Kidney Disease Improving Global Outcome - KDI$\mathrm{GO})^{5,6}$, ha propuesto recientemente una definición formal para la ERC. Esta terminología ha permitido estandarizar las comunicaciones médicas, tanto clínicas como epidemiológicas, y también hacerla más comprensible a los pacientes. Esta definición ha facilitado los estudios de mapeo basados en poblaciones, estimulando la prevención, tratamiento oportuno y calidad de la atención de la $\mathrm{ERC}^{6}$.

Las guías de la KDIGO apoyan el uso de la expresión "enfermedad renal crónica" (ERC) para referirse a todo el espectro de la enfermedad que ocurre luego del inicio del daño renal. La severidad del síndrome resultante está representado en un esquema de etapificación que se extiende desde el daño renal con función conservada (fase 1) hasta el nivel de insuficiencia renal que requiere tratamiento de sustitución renal (fase 5) (Tablas 1 y 2).

La ERC se define como la presencia de un daño renal estructural con VFG normal o levemente reducida (VFG 60-90 mL), independientemente de la etiología subyacente (Tabla 1). La evidencia del daño estructural potencialmente progresivo puede derivar de un estudio histológico o imagenológico, 0 de las alteraciones persistentes del examen de orina por un plazo superior a tres meses, particularmente la presencia de albuminuria.

De acuerdo al KDIGO, una VFG inferior a $60 \mathrm{~mL}$ corresponde a una ERC, sin requerir evidencia adicional de daño renal estructural. Este punto de corte fue seleccionado debido a que representa el $50 \%$ o más de reducción de la función renal normal

\section{Tabla 1. D efinición de enfermedad renal crónica}

1. Daño renal por $\geq 3$ meses, definido por anormalidades estructurales o funcionales del riñón, con 0 sin disminución de la VFG, manifestado por:

- Anormalidades anátomo o histopatológicas, o

- Marcadores de daño renal, incluyendo anormalidades sanguíneas o urinarias, o anormalidades en exámenes imagenológicos.

2. VFG $<60 \mathrm{~mL} / \mathrm{min} / 1,73 \mathrm{~m}^{2}$ por $\geq 3$ meses, con o sin daño renal.

Tomado de National Kidney Foundation KD: Clinical Practice Guidelines for Chronic Kidney Disease: Evaluation, Classification, and Stratification. Am J Kidney Dis 2002; 39 (suppl 1): S1-S266.

Tabla 2. Fases y prevalencia de la enfermedad renal crónica (edad >20 años)

\begin{tabular}{|llcc|}
\hline Fase & Descripción & VFG $\left(\mathrm{mL} / \mathrm{min} / 1,73 \mathrm{~m}^{2}\right)$ & $\%$ \\
\hline 1 & Daño renal con VFG normal o $\uparrow$ & $\geq 90$ & 3,3 \\
2 & Daño renal con $\downarrow$ leve de la VFG & $60-89$ & 3,0 \\
3 & $\downarrow$ moderada de la VFG & $30-59$ & 4,3 \\
4 & $\downarrow$ severa de la VFG & $15-29$ & 0,2 \\
5 & Insuficiencia renal & $<15$ o diálisis & 0,1 \\
\hline
\end{tabular}

Tomado de National Kidney Foundation KD: Clinical Practice Guidelines for Chronic Kidney Disease: Evaluation, Classification, and Stratification. Am J Kidney Dis 2002; 39 (suppl 1): S1-S266. 
de un adulto joven, además de la evidencia que demuestra que la morbimortalidad aumenta a medida que la VFG disminuye bajo $60 \mathrm{~mL}$ Los pacientes con VFG entre 60 y 89, sin daño estructural, no están definidos como portadores de $\mathrm{ERC}^{5,6}$.

La determinación de VFG no precisa de medición de velocidad de depuración de creatinina de 24 $h$, y se recomienda el uso de la VFG estimada de acuerdo a la fórmula de Cockcroft-Gault o la fórmula del MDRD (Estudio de Modificación de la Dieta en Enfermedades Renales) ${ }^{7}$. Para optimizar dicha VFG estimada existen iniciativas para la estandarización de la medición de creatinina sérica a nivel global.

\section{MECANISMOS DE PROGRESIÓN EN LA ENFERMEDAD RENAL CRÓNICA}

En la práctica clínica, la mayoría de las nefropatías progresan lentamente hacia la pérdida definitiva de la función renal, sin embargo, algunas enfermedades renales tienen una rápida evolución a la insuficiencia renal crónica terminal, pero esto es un hecho raro de observar.

En 1952, R. Platt observó que en ratas, a las cuales se les removía el $80 \%$ de la masa renal, se producía hipertrofia de las nefronas remanentes, interpretando este hallazgo como un mecanismo adaptativo para compensar la pérdida funcional ${ }^{8}$. Treinta años después, en 1982, B. Brenner y cols, introdujeron el concepto de que la naturaleza progresiva de la pérdida funcional renal es el resultado de cambios hemodinámicos compensatorios a nivel glomenular, fundamentalmente hipertensión e hiperfiltración glomerular en las nefronas remanentes. Estos cambios hemodinámicos causan daño sostenido en dichas nefronas 9 . Posteriormente, en 1986, T. Bertani y cols, destacaron la importancia del daño tubulointersticial asociado a la proteinuria, suginiendo que las proteínas filtradas a través del capilar glomerular pueden tener toxicidad renal intrínseca y contribuyen a la progresión del daño renal ${ }^{10}$.

Desde la publicación de los estudios de Brenner, los nefrólogos han concordado en definir la progresión de las enfermedades renales como la pérdida progresiva de la VFG a una velocidad mayor que la fisiológica, que correspondería a 1 mL por año, en adultos mayores de 40 años. Este deterioro progresivo se asocia a un reemplazo del tejido renal por tejido fibroso a diferentes niveles, involucrando una progresiva glomeruloesclerosis, nefroesclerosis y fibrosis túbulo-intersticial.

La pérdida nefronal, independientemente de su etiología, provoca respuestas adaptativas en las nefronas remanentes que conllevan hipertensión e hiperfiltración glomenular, paso de proteínas al espacio uninario con proteinuria, activación intramenal del sistema renina angiotensina (SRA), activación tubular con compromiso túbulo-intersticial, transdiferenciación de células epiteliales tubulares a miofibroblastos y finalmente fibrosis del parénquima renal con pérdida definitiva de la función (Figuras 2 y 3).

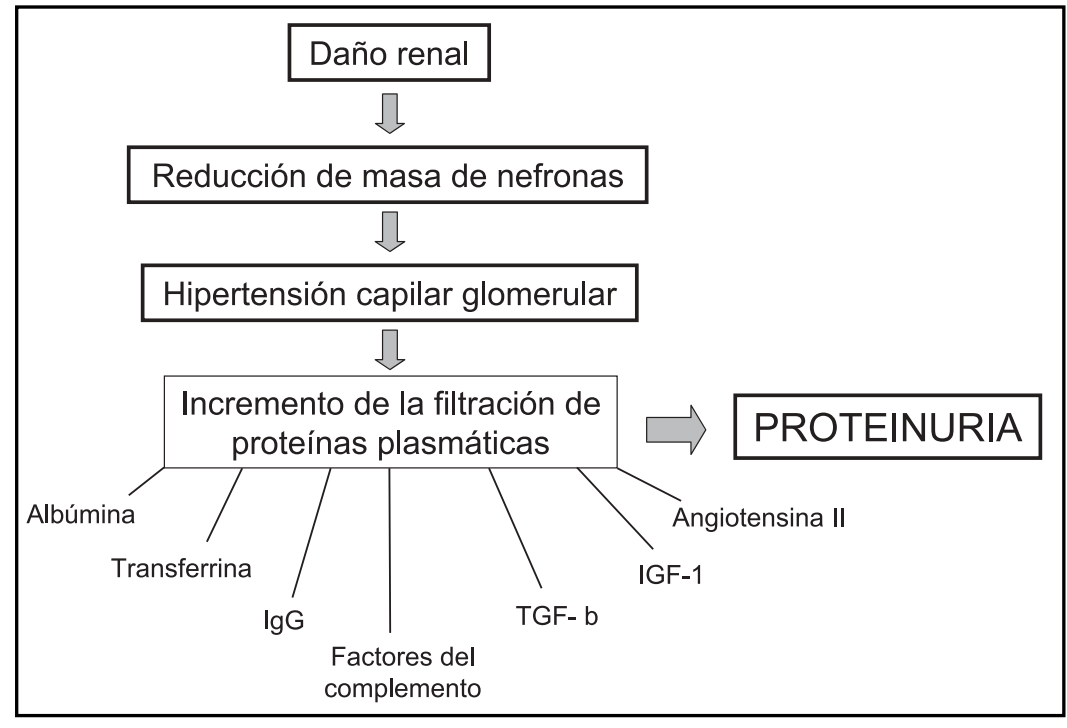

FIgURA 2. Consecuencias de la reducción de masa nefronal y sus mecanismos adaptativos. 


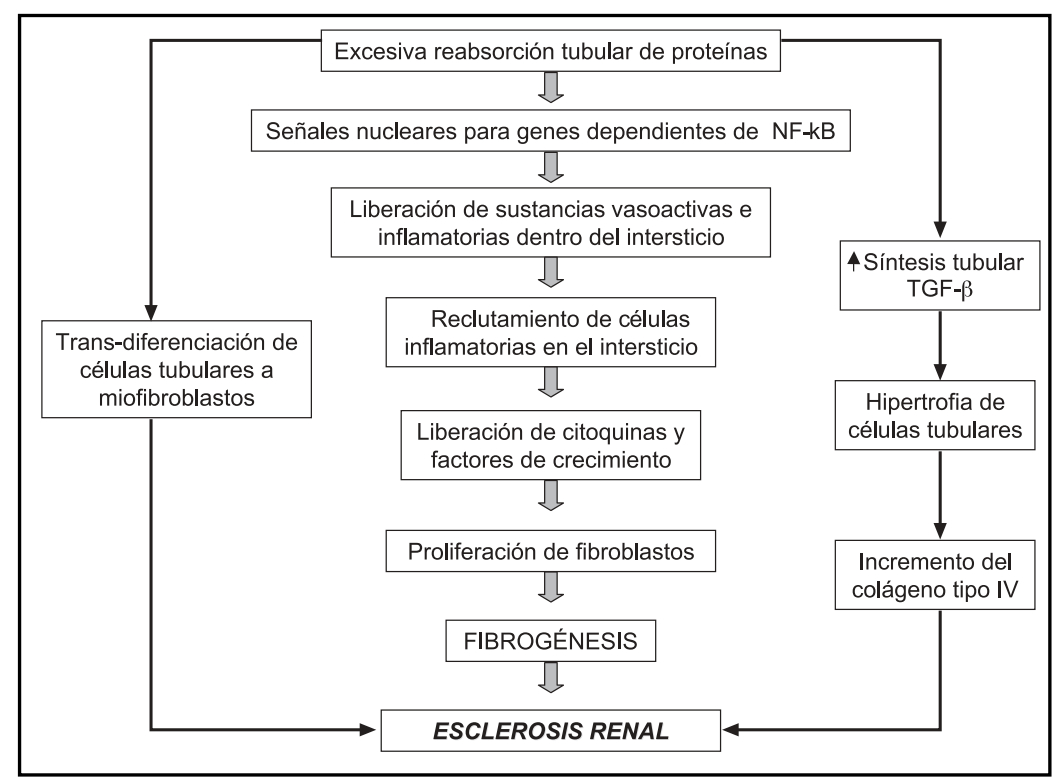

FIgURA 3. Mecanismos activados por el tráfico tubular de proteínas que conducen a la esclerosis renal.

Aparentemente, la glomeruloesclerosis puede evolucionar en etapas, con un daño e inflamación endotelial inicial, seguido de proliferación y activación mesangial, y una etapa final de esclerosis y fibrosis. En estas etapas patogénicas se ha observado una similitud con el daño aterosclerótico de grandes vasos ${ }^{11}$.

Por otra parte, la fibrosis túbulo-intersticial juega también un rol clave en la progresión de la ERC y es así como la severidad de los cambios túbulo-intersticiales se correlacionan mejor con la pérdida de función renal que la presencia de glomeruloesclerosis.

Basados en numerosas evidencias experimentales, los factores más importantes de daño túbulo-intersticial son la presencia y magnitud de la proteinuria y la activación del SRA.

En nuestro laboratorio, hemos obtenido evidencia directa de la participación de los mecanismos mencionados en nefropatías progresivas en humanos, en pacientes diabéticos y en no diabéticos.

La proteinuria persistente influye en la progresión de las enfermedades glomerulares determinando una activación tubular de los factores de transcripción nuclear kappa B (NF-кB) y proteína activada 1 (AP-1), los que participan en la transcripción de genes proinflamatorios (MCP-1, RANTES, osteopontina) y genes profibrogénicos (TGF-ß, PDGF), conducentes a una reacción inflamatoria y fibrosis del intersticio ${ }^{12}$ (Figura 4). El infiltrado inflamatorio compuesto, fundamentalmente, de linfocitos CD4, CD8 y monocito/macrófagos CD68, puede, además, participar en la génesis de la hipertensión sal-sensible y en la retención hidrosalina ${ }^{13,14}$. La generación de colágeno, elemento central en la fibrosis renal, deriva en un porcentaje importante de la transdiferenciación o cambio fenotípico de las células epiteliales tubulares en células mesenquimáticas miofibroblásticas, siendo el TGF-ß el principal inductor de esta transdiferenciación.

Por otra parte, la angiotensina II (Ag II) tiene un rol clave en la progresión de la enfermedad renal. Participa en los cambios hemodinámicos glomerulares y en los cambios de permeabilidad de la barrera de filtración glomerular, contribuyendo a incrementar la proteinuria ${ }^{15}$. Adicionalmente, la $\mathrm{Ag}$ II se ha involucrado directamente en la génesis del daño túbulo-intersticial y en la fibrosis ${ }^{16}$, comportándose como una genuina citoquina pro-fibrótica y pro-inflamatoria ${ }^{17}$. La activación intrarrenal del SRA parece desempeñar un rol fundamental en estos procesos y además la proteinuria per se puede activar la generación de Ag II intrarrenal, perpetuando dicha activación. También se ha observado que la Ag II puede inducir activación del factor de transcripción NF-кB en células tubulares renales y así participar como citoquina pro-inflamatoria.

En nefropatía membranosa idiopática, en la que un número importante de casos progresa a la pérdida definitiva de la función renal asociada a 
una proteinuria persistente, hemos observado una activación selectiva del SRA intrarrenal con una sobreexpresión tubular de enzima convertidora de angiotensina (ECA) a nivel del ribete en cepillo de los túbulos proximales, y una neo-inducción de ECA a nivel de células miofibroblásticas intersticiales, particularmente en sitios de daño túbulo- intersticial (Figura 5). Paralelamente, hemos evidenciado expresión de Ag II en células tubulares y células infiltrantes inflamatorias ${ }^{18,19}$.

Hallazgos similares, con una marcada activación intrarrenal del SRA, y neo-inducción de ECA se ha observado en pacientes diabéticos macroalbuminúri$\cos ^{20}$ (Figura 5). Conjuntamente en estos mismos
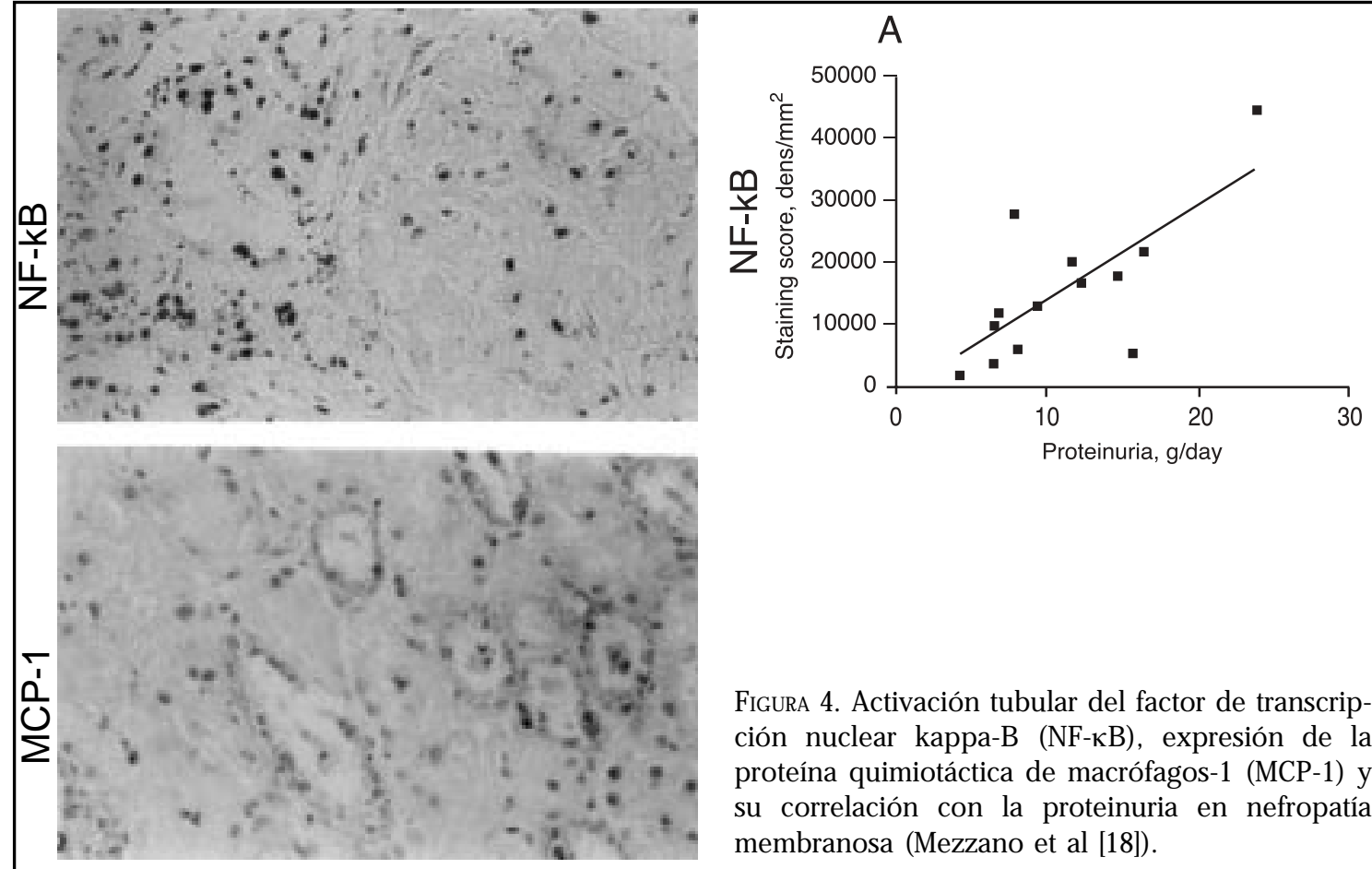

FiguRa 4. Activación tubular del factor de transcripción nuclear kappa-B (NF-кB), expresión de la proteína quimiotáctica de macrófagos-1 (MCP-1) y su correlación con la proteinuria en nefropatía membranosa (Mezzano et al [18]).

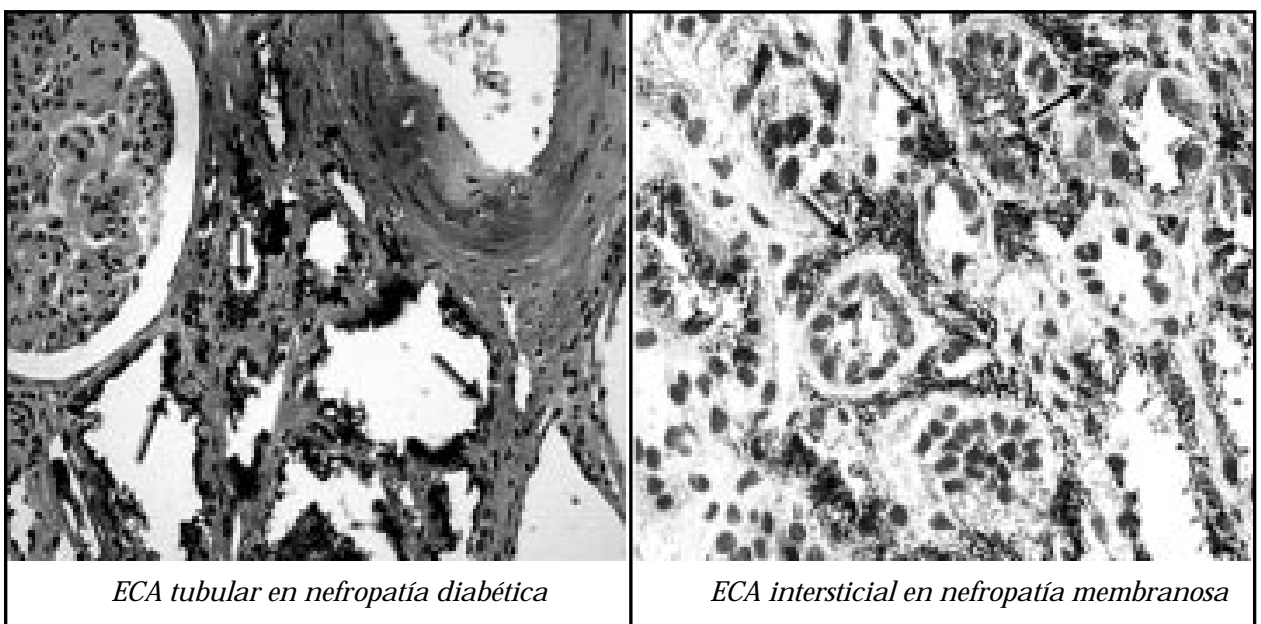

FIGURA 5. Sobreexpresión tubular en el ribete en cepillo y neo-inducción intersticial de enzima convertidora de angiotensina en nefropatía diabética y nefropatía membranosa (Mezzano et al [19-20]). 
pacientes también hemos observado activación tubular de NF-кB, sobreexpresión de citoquinas proinflamatorias (MCP-1, RANTES, osteopontina) e infiltrado inflamatorio túbulo-intersticial, confirmando que la proteinuria y la activación del SRA intrarrenal son los principales agentes involucrados en el daño túbulo-intersticial y por ende en la progresión de la nefropatía diabética ${ }^{20,21}$ (Figura 6).

\section{INTERVENCIONES PARA RETARDAR LA PROGRESIÓN DE LA ENFERMEDAD RENAL CRÓNICA}

En nefropatías progresivas se ha establecido una fuerte comelación entre la magnitud del control de la presión arterial y la velocidad de declinación de la función renal: a mejor control de la presión arterial, mejor es la preservación de la filtración glomerular. Es así como el tratamiento de la hipertensión sistémica fue la primera intervención que demostró reducir en forma significativa la velocidad de progresión de la ERC siendo aún el eje central de las estrategias de renoprotección. Esto ha sido ampliamente demostrado en numerosos estudios en diabéticos tipo 1 y 2 y en no diabéticos con ERC ${ }^{22,23}$. También la proteinuria es un predictor pronóstico renal fuerte e independiente ya que al reducir la proteinuria se observa una menor velocidad de declinación de la función renal. En el caso de la diabetes, la proteinuria en etapa de microalbuminuria (de 20-200 $\mathrm{mg} / \mathrm{min}$ o $30-300 \mathrm{mg} /$ día) constituye el signo más precoz de apanición de la nefropatía diabética y de ahí la importancia de su pesquisa de tal forma de intervenir precozmente sobre el daño renal en curso. Cuando aparece la macroalbuminuria (>200 $\mu \mathrm{g} / \mathrm{min} 0>300 \mathrm{mg} /$ día) y se eleva la presión arterial, se produce el compromiso progresivo de la función renal. Por otra parte, la microalbuminuria y la albuminuria no sólo representan el daño renal incipiente o establecido y son de utilidad en predecir la evolución sino que además en múltiples estudios se han asociado a un incremento significativo del niesgo cardiovascular, lo que también ha sido demostrado para nefropatías no diabéticas ${ }^{24}$.

Así se ha instituido el concepto de renoprotección, siendo las principales medidas recomendadas el control farmacológico de la presión arterial y la reducción de la proteinuria ${ }^{25}$.

Estudios multicéntricos en nefropatías diabética y no diabética han demostrado la eficacia de algunas intervenciones terapéuticas. El control metabólico estricto en la diabetes tipo 1 y 2 ha revelado ser útil en reducir las complicaciones microvasculares, entre las cuales se encuentra la nefropatía ${ }^{26,27}$. El bloqueo del SRA mediante inhibidores de la enzima convertidora de angiotensina (IECAs) se ha demostrado útil en el control de la presión arterial y en la reducción de la proteinuria; sin embargo, estos fármacos, a igual control de presión arterial, son capaces de reducir la proteinuria más efectivamente que los antihipertensivos convencionales ${ }^{28}$.

En enfermedad renal crónica no diabética, se ha acumulado una gran cantidad de evidencia expenmental y clínica, sobre la eficacia del bloqueo del SRA mediante el uso de IECAs o antagonistas de receptor

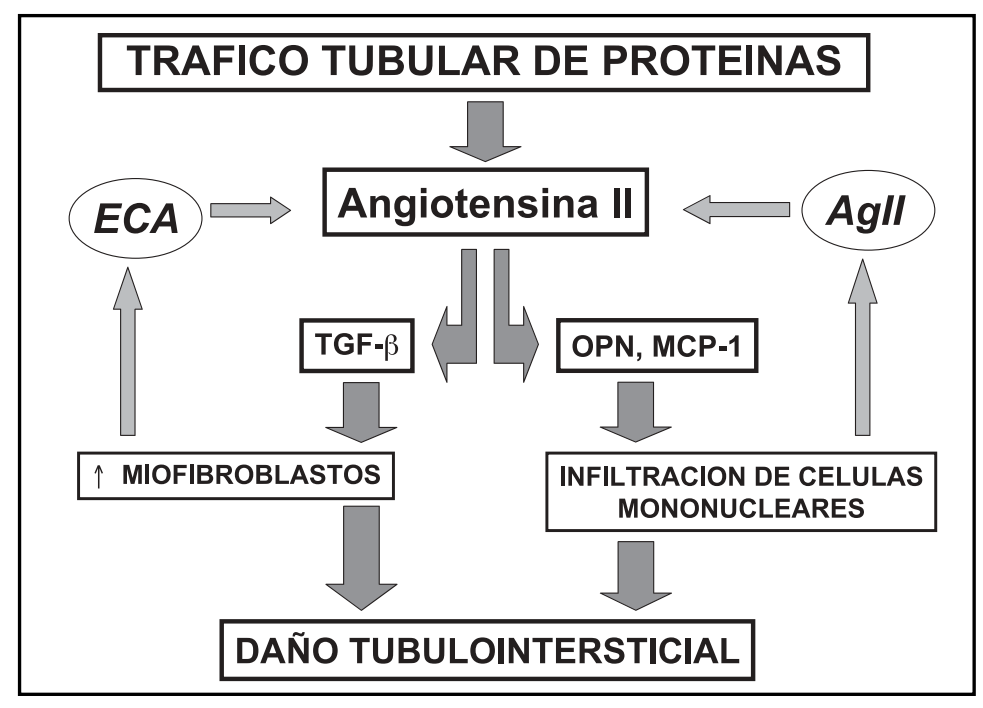

FiguRa 6. Tráfico tubular de proteínas, activación del sistema renina angiotensina intrarrenal y respuestas pro-inflamatorias y profibrogénicas (Mezzano et al [19-20]). 
de Ag tipo 1 (ARA) en retardar o prevenir la progresión de la enfermedad renal crónica ${ }^{28-31}$. En el estudio AIPRI, mediante el uso de benazepril se observó una reducción de $53 \%$ en el riesgo de duplicar la creatininemia basal, sin embargo, debido a que los niveles de presión arterial logrados no fueron iguales entre el grupo de tratamiento y el grupo control, no se pudo atribuir todo el efecto benéfico a un efecto específico de la inhibición del SRA independientemente de la reducción de la presión arterial ${ }^{30}$. En el estudio REIN (Ramipril efficacy in nephropathies) realizado en pacientes con nefropatías proteinúricas no diabéticas, los niveles de control de presión arterial fueron similares entre los grupos, observándose un deterioro de la función renal significativamente menor en el grupo que usó ramipril, especialmente en los pacientes que en condiciones basales presentaban una proteinuria mayor de 3 gramos o una función renal más reducida ${ }^{31}$. Un metaanálisis (estudio AIPRID) que evaluó 11 tratamientos randomizados y controlados con un total de 1.860 pacientes, 938 con IECAs y con un seguimiento promedio de 2,2 años, evidenció que la inhibición del SRA redujo el niesgo de duplicar la creatininemia basal o de llegar a una ERC fase 5 en $30 \%$ (riesgo relativo 0,7) ${ }^{32}$.

Los IECAs también han demostrado utilidad en nefroesclerosis hipertensiva, especialmente en los pacientes que presentan un grado mínimo de proteinuria (estudio AASK) ${ }^{33}$. En enfermedad renal poliquística avanzada, estudios en curso han mostrado algunas evidencias de que los IECAs son más efectivos en reducir la proteinuria, siendo mayor el beneficio en aquellos pacientes con mayor proteinuria basal. Finalmente, se ha propuesto el uso de la asociación de IECAs con ARA con el fin de lograr un bloqueo más completo del SRA. Varios estudios pequeños habían mostrado su beneficio en cuanto a una mayor reducción de la proteinuria y de la presión arterial ${ }^{34}$, sin embargo, el estudio COOPERATE confirmó que el efecto protector renal también es superior con respecto a la monoterapia ${ }^{35}$.

En nefropatía diabética, la inhibición del SRA había demostrado su utilidad en diabetes tipo 1 con el uso de captopril, el cual reduce la progresión de nefropatía incipiente a nefropatía establecida $^{29}$. Sin embargo, sólo recientemente se ha demostrado la utilidad de los ARA en diabéticos tipo 2, en prevenir la progresión de nefropatía incipiente a nefropatía establecida y en retardar el deterioro de la función renal en los pacientes con una nefropatía diabética ya establecida (macroalbuminúricos) ${ }^{36-38}$. En estos estudios no se comparó el efecto renoprotector de los ARA con el de los IECAs, pero recientemente se publicó otro estudio en el que se comparó el tratamiento con telmisartán o enalapril en 254 diabéticos tipo 2 con nefropatía incipiente. Se evaluó prospectivamente la reducción de la velocidad de filtración glomerular a 5 años, observándose que telmisartán no fue inferior a enalapril. Tampoco se observaron diferencias en relación a la variación de la creatininemia, albuminuria, incidencia de ERC fase 50 eventos cardiovasculares, lo que permitiría concluir que ambos fármacos tienen eficacia similar ${ }^{39}$.

¿Es posible efectuar prevención primaria, es decir evitar la aparición de microalbuminuria, en el diabético tipo 2 mediante la inhibición del SRA? El estudio BENEDICT, publicado recientemente, demostró que el uso de trandolapril en diabéticos tipo 2 con hipertensión arterial es capaz de reducir el riesgo de presentar microalbuminuria en $40 \%$ con respecto a placebo 40 .

Basándose en estudios clínicos de intervención, observacionales y epidemiológicos, se han reconocido otros factores que pueden incidir en la progresión. El exceso de ingesta de proteínas, la hiperlipidemia y el tabaquismo aceleran la pérdida de función renal por lo que la reducción en las proteínas de la dieta ${ }^{41}$, el control de la hiperlipidemia $^{42}$ y la suspensión del tabaco ${ }^{43}$ se han considerado parte integral del protocolo de manejo multifactorial de prevención de la progresión de la enfermedad renal crónica.

La Tabla 3 resume los principales estudios randomizados de intervención en relación a la prevención de la progresión.

\section{RECOMENDACIONES}

En base a la evidencia mostrada anteriormente, en los últimos años se han desarrollado recomendaciones sobre las cuales cada vez hay más consenso.

En nefropatías diabéticas como no diabéticas se aplican las siguientes recomendaciones:

1. El control de la hipertensión arterial debe ser estricto con valores menores a $130 / 80 \mathrm{mmHg}$. $\mathrm{Si}$ existe proteinuria los valores deben ser menores a $125 / 75 \mathrm{mmHg}$. 
2. La inhibición del SRA es otra intervención fundamental, especialmente en pacientes proteinúricos, con el claro objetivo, además de controlar la presión arterial, de reducir la proteinuria a valores menores a $300 \mathrm{mg} /$ día.
Estudios en desarrollo sugieren que el tratamiento combinado con IECAs y ARAs estaría indicado cuando con monoterapia y un óptimo control de la hipertensión, el paciente persiste con proteinuria mayor de 1 gramo/día.

\section{Tabla 3. Algunos estudios randomizados que han demostrado eficacia del bloqueo del sistema renina-angiotensina para reducir la progresión de la enfermedad renal crónica}

Nefropatías no diabéticas:

- Estudio de inhibición de la enzima convertidora de angiotensina en la insuficiencia renal progresiva (AIPRI) (Ref 30).

- Estudio de eficacia del ramipril en nefropatía (REIN) (Ref 31).

- Estudio de inhibición de la ECA en la enfermedad renal progresiva (AIPRID) (Ref 32).

- Estudio de hipertensión y enfermedad renal en afroamericanos (AASK) (Ref 33).

- Estudio de tratamiento combinado con IECAs y ARA en enfermedad renal no diabética (COOPERATE) (Ref 35).

Nefropatías diabética:

- Estudio de evaluación y prevención de eventos cardíacos (HOPE, MICRO-HOPE).

- Estudio reducción de eventos en DMNID con el antagonista de angiotensina II losartán (RENAAL) (Ref 37).

- Estudio del irbesartán en pacientes con DM 2 y microalbuminuria (Ref 38).

- Estudio del irbesartán en nefropatía diabética (IDNT) (Ref 36).

- Estudio de prevención de la microalbuminuria en DM 2 (BENEDICT) (Ref 40).

- Estudio de bloqueadores de receptor de AT II versus IECAS en nefropatía de la DM2 (Ref 39).

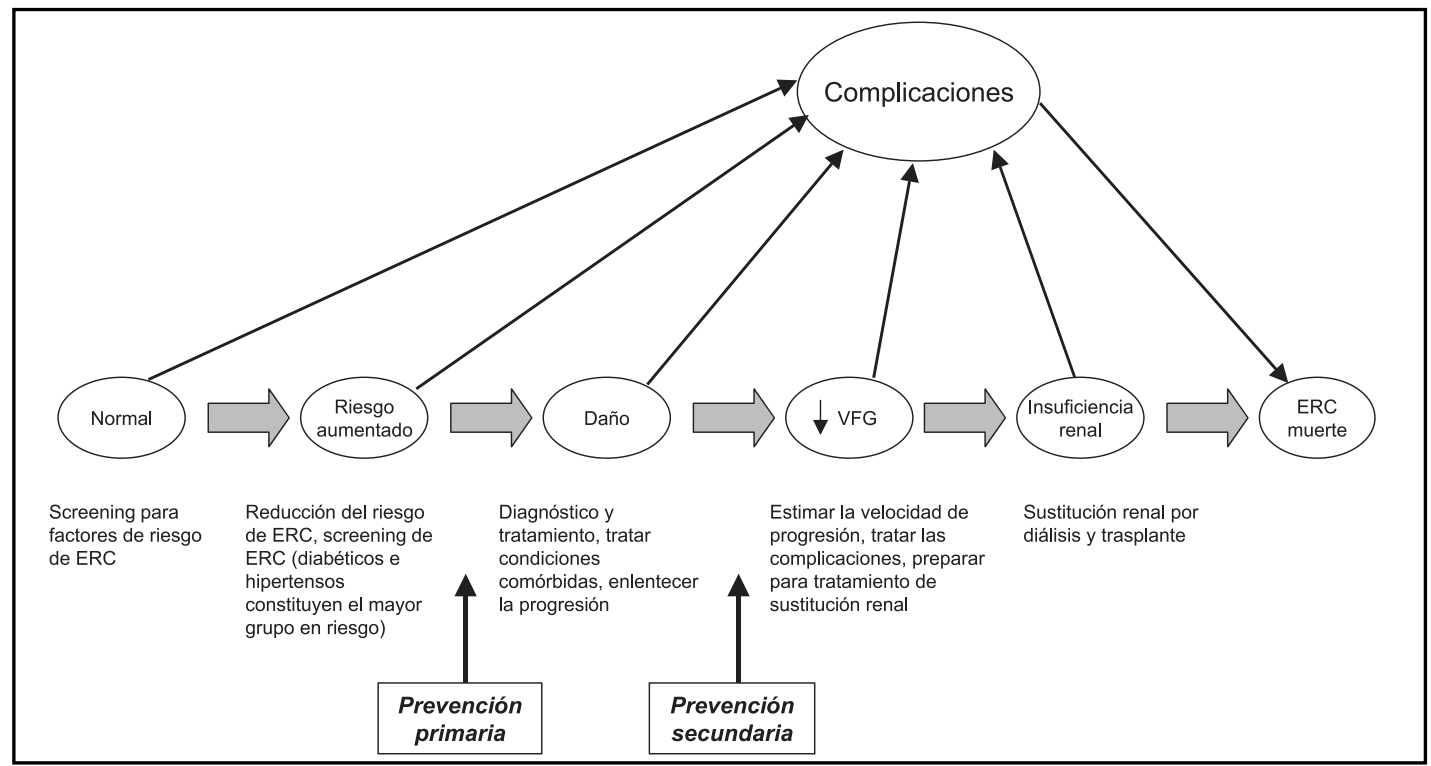

FiguRA 7. Manejo clínico de la enfermedad renal crónica en sus diferentes fases. Tomado de National Kidney Foundation KD: Clinical Practice Guidelines for Chronic Kidney Disease: Evaluation, Classification and Stratification. Am J Kidney Dis. 2002, 39: S1-S6. 
3. Tratar la dislipidemia, alcanzando valores de LDL inferiores a $100 \mathrm{mg} / \mathrm{dL}(2,6 \mathrm{mmol} / \mathrm{L})$ tanto para reducir el riesgo de progresión como el riesgo de eventos cardiovasculares.

4. La reducción de la ingesta proteica a valores de 0,6 a $0,8 \mathrm{~g} / \mathrm{kg} /$ día de proteínas de alto valor biológico es otra medida que puede ser útil.

5. Si bien no existen estudios de intervención respecto a la suspensión del tabaco, ésta podría ser una de las medidas de más alto impacto en reducir la progresión, además de

\section{REFERENCIAS}

1. U.S. Renal Data System. USRDS 2003 Annual data Report: Atlas of end-stage renal disease in the Unites States. Bethesda, MD, National Institutes of Health, 2003, pp 1-560.

2. Мrтch WE. Treating diabetic nephropathy - Are there only economic issues? N Engl J Med 2004; 351: 1934-6.

3. Go SA, Chertow GM, Fan D, MC CuLoch CE, Hsu $\mathrm{CH}-\mathrm{Y}$. Chronic kidney disease and the risks of death, cardiovascular events and hospitalization. N Engl J Med 2004; 351: 1296-305.

4. Encuesta Nacional de Salud 2003. Disponible en http:/ /www.minsal.cl/ici/destacados/Folleto\%20FINALpdf

5. Eknoyan G, Lameire N, Barsoum R, Eckardt K-U, LeVIN A, LeVIN N ET aL. The burden of kidney diseases: Improving global outcomes. Kidney Int 2004; 66: 1310-4.

6. National Kidney Foundation KD. Clinical Practice Guidelines for Chronic Kidney Disease: Evaluation, Classification, and Stratification. Am J Kidney Dis 2002; 39 (suppl 1): S1-S266.

7. LeVEY AS. Nondiabetic kidney disease. N Engl J Med 2002; 347: 1505-11.

8. Piatt R. Structural and functional adaptation en renal failure. BMJ 1952; i; 1372-7.

9. BRENNER BM, MEYer TW, HostetTER TH. Dietary protein intake and the progressive nature of kidney disease: the role of hemodinamically mediated glomenular injury in the pathogenesis of progressive glomerular sclerosis in aging, renal ablation, and intrinsec renal disease. N Engl J Med 1982; 307: 652-9.

10. Bertani T, CutiLo F, Zoja C, Broggini M, Remuzzi G. Tubulointerstitial lesions mediate renal damage in adriamycin glomerulopathy. Kidney Int 1986; 30: 488-96. su efecto benéfico en reducir significativamente los eventos cardiovasculares.

6. En el caso de la diabetes, el objetivo es la euglicemia, recomendándose valores de hemoglobina glicosilada normales (menores de 7\%).

7. Otras medidas que podrían ser de utilidad son el control del peso corporal, realizar ejercicio físico y reducir la ingesta de sal y alcohol.

Las recomendaciones para el manejo clínico de la ERC en sus diferentes fases se resumen en la Figura 7.

11. Fogo AB. Progression and potential regression of glomerulosclerosis. Kidney Int 2001; 50: 804-19.

12. Mezzano SA, Ruiz-Ortega M, Egido J. Angiotensin II and renal fibrosis. Hypertension 2001; 38: 635-8.

13. Rodríguez-Iturbe B, Herrera-Acosta J, Johnson RJ. Interstitial inflammation, sodium retention, and the pathogenesis of nephrotic edema: a unifying hypothesis. Kidney Int 2002; 62: 1379-84.

14. Johnson RJ, HerRera-Acosta J, Schreiner GF, RodríGUEz-ITURBe B. Subtle acquired renal injury as a mechanism of salt-sensitive hypertension. $\mathrm{N}$ Engl J Med 2002; 346: 913-23.

15. Ruggenenti P, Aros C, Remuzzi G. Renin-angiotensin system, proteinuria, and tubulointerstitial damage. Contrib Nephrol 2001; 135: 187-99.

16. Mezzano SA, Ruzz-Ortega M, Egido J. Angiotensin II and renal fibrosis. Hypertension 2001; 38: 635-8.

17. Ruiz-Ortega M, Ruperez M, Lorenzo O, Esteban V, Blanco J, Mezzano $S$ et al. Angiotensin II regulates the synthesis of proinflammatory cytokines and chemokines in the kidney. Kidney Int 2002; [Suppl] 82: S12-S22.

18. Mezzano SA, Droguett MA, Burgos ME, Ardiles LG, Aros CA, CAORSI I ET al. Overexpression of chemokines, fibrogenic cytokines, and myofibroblasts in human membranous nephropathy. Kidney Int 2000; 57: 147-58.

19. Mezzano SA, Aros CA, Droguett A, Burgos ME, Ardiles LG, Flores CA et al. Renal angiotensin II up-regulation and myofibroblast activation in human membranous nephropathy. Kidney Int 2003; [Suppl] 86: S39-S45.

20. Mezzano S, Droguett A, Burgos ME, Ardiles LG, FLoRES CA, ARos CA ET AL. Renin-angiotensin system activation and interstitial inflammation in human diabetic nephropathy. Kidney Int 2003; [Suppl] 86: S64-S70. 
21. Mezzano S, Aros C, Droguett A, Burgos ME, Ardiles L, FLORES C ET AL. NF-kappaB activation and overexpression of regulated genes in human diabetic nephropathy. Nephrol Dial Transplant 2004; 19: 2505-12.

22. Parving HH, Andersen AR, Smidt UM, Svendsen PA. Early aggressive antihypertensive treatment reduces rate of decline in kidney function in diabetic nephropathy. Lancet 1983; 8335: 1175-9.

23. Kes P, RatKovic-Gusic I. The role of arterial hypertension in progression of renal failure. Kidney Int 1996; [Suppl] 55: S72-S74.

24. De ZeEuw D. Albuminuria, not only a cardiovascular/renal risk marker, but also a target for treatment? Kidney Int 2004; 66: S2-S6.

25. Peterson JC, Adier S, Burkart JM, Greene T, Hebert LA, HunsiKer LG ET AL. Blood pressure control, proteinuria, and the progression of renal disease: the Modification of Diet in Renal Disease Study. Ann Intern Med 1995; 123: 754-62.

26. The Diabetes Control and Complications (DCCT) ReSEARCH Group. Effect of intensive therapy on the development and progression of diabetic nephropathy in the Diabetes Control and Complications Trial. Kidney Int 1995; 47: 1703-20.

27. UK Prospective Diabetes Study (UKPDS) Group. Intensive blood glucose control with sulphonylureas or insulin compared with conventional treatment and risk of complications in patients with type 2 diabetes (UKPDS 33). Lancet 1998; 352: 837-53.

28. Aros C, ReMUZZI G. The renin-angiotensin system in progression, remission and regression of chronic nephropathies. J Hypertens 2002; 20 [Suppl 3]: S45-S53.

29. Lewis EJ, Hunsicker LG, Bain RP, Rohde RD. The effect of angiotensin-converting-enzyme inhibition on diabetic nephropathy. N Engl J Med 1993; 329: $1456-62$.

30. Maschio G, Alberti D, Janin G, Locatemi F, Mann JFE, MотоLESE M ET AL. Effect of the angiotensinconverting-enzyme inhibitor benazepril on the progression of chronic renal insufficiency. N Engl J Med 1996; 334: 939-45.

31. The Gisen Group. Randomized placebo-controlled trial of effect of ramipril on decline in glomerular filtration rate and risk of terminal renal failure in proteinuric, non-diabetic nephropathy. Lancet 1997; 349: 1857-63.

32. Jafar TH, Schmid CH, Landa M, Giatras I, Toto R, REMUZZi G ET al. Angiotensin-converting enzyme inhibitors and progression of nondiabetic renal disease: A meta-analysis of patient-level data. Ann Intern Med 2001; 135: 73-87.
33. Agodoa L, Appel L, Bakris G, Beck G, Bourgoignie J, BRIGGS JP ET AL. Effect of ramipril vs amlodipine on renal outcomes in hypertensive nephrosclerosis. JAMA 2001; 285: 2719-28.

34. Campbell R, Sangaul F, Perticucci E, Aros C, Viscarra C, Perna A et al. Effects of combined ACE inhibitor and angiotensin II antagonist treatment in human chronic nephropathies. Kidney Int 2003; 63: 1094-103.

35. Nakao N, Yoshimura A, Morita H, Takada M, Kayano T, IDEuRA T. Combination treatment of angiotensin-II receptor blocker and angiotensinconverting-enzyme inhibitor in non-diabetic renal disease (COOPERATE): A randomised controlled trial. Lancet 2003; 361: 117-24.

36. Lewis EJ, Hunsicker LG, Ciarke WR, Berl T, Pohl MA, LEwIS JB ET AL. Renoprotective effect of the angiotensin-receptor antagonist irbesartan in patients with nephropathy due to type 2 diabetes. $\mathrm{N}$ Engl J Med 2001; 345: 851-60.

37. Brenner BM, Cooper ME, De Zeeuw D, Keane WF, Mitch WE, Parving HH et aL. Effects of losartan on renal and cardiovascular outcomes in patients with type 2 diabetes and nephropathy. N Engl J Med 2001; 345: 861-9.

38. Parning H-H, Lehnert H, Brochner-Mortensen J, Gomis R, ANDERSEN R, ARNER P ET AL. The effect of irbesartan on the development of diabetic nephropathy in patients with type 2 diabetes. NEngl J Med 2001; 345: 870-8.

39. Barnett AH, Bain SC, Bouter P, Karlberg B, Madsbad S, Jervell J et al. Angiotensin-receptor blockade versus converting-enzyme inhibition in type 2 diabetes and nephropathy. N Engl J Med 2004; 351: 1952-61.

40. Ruggenenti P, Fassi A, Parvanova-Iueva A, Bruno S, Petrov-Iuev I, Brusegan V et al for the Bergamo Nephrologic Diabetes Complications Trial (BENEDICT) investigators. Preventing microalbuminuria in type 2 diabetes. N Engl J Med 2004; 351: 1941-51.

41 Kiahr S, Levey AS, Beck GJ, Caggiula AW, HunsicKER L, KUSEK JW ET AL. The effects of dietary protein restriction and blood pressure control on the progression of chronic renal disease. N Engl J Med 1994: 330: 877-84.

42. Samuelsson O, Mules $H$, Knight-Gibson C, Attman PO, KRON B, LaRSSON R ET al. Lipoprotein abnormalities are associated with increased rate of progression of human chronic renal insufficiency. Nephrol Dial Transplant 1997; 12: 1908-15.

43. RemuZZi G. Cigarette smoking and renal function impairment. Am J Kidney Dis 1999; 33: 807-13. 